(C)2009 IEEE. Personal use of this material is permitted. However, permission to reprint/republish this material for advertising or promotional purposes or for creating new collective works for resale or redistribution to servers or lists, or to reuse any copyrighted component of this work in other works must be obtained from the IEEE. 


\title{
Assistive Technology Interfaces For The Blind
}

\author{
David J. Calder \\ Curtin University of Technology \\ Bentley, Perth, \\ West Australia \\ Tel. 61-8-9266 2875 \\ david.calder@cbs.curtin.edu.au
}

\begin{abstract}
Assistive technology devices for the blind are portable electronic devices that are either hand-held or worn by the visually impaired user, to warn of obstacles ahead. Many assistive technology devices use ultrasonic pulse-echo techniques to gauge subject to object distance. Some use infrared light transceivers or laser technology to locate and warn of obstacles. These devices exhibit a number of problems, the most significant of which are related to the interface display that conveys navigation/obstacle warning information to the user. Other sensory channels should not be compromised by the device. This is exactly what can happen when, for example, audio signals are used in obstacle warning on/off displays or more significantly in orientation solutions, where continuous streams of synthetically generated stereo sound mask the natural ambient sound cues used by the blind. Despite the challenges, the commendable feature all these assistive device developers have in common is; they are striving to help a section of the population with a severe disability. Even if there is only partial success in this endevour to assist the blind, the small companies that produce these devices all have the right motive. That is a big step in the right direction. The author has attempted to address some of the problems mentioned in this paper by producing a first working prototype. Improvements to this original design form the basis for ongoing prototype development within the DEBI Institute at Curtin University.
\end{abstract}

Keywords - Obstacle warning displays, assistive technology, sound interface displays, laser, disabled, infrared, long cane, portable electronic device, sensory channels, visually impaired, ultrasonic pulse-echo, ambient sound cues.

\section{INTRODUCTION}

$\mathrm{T}$ here are approximately ten competing mobility aids and orientation mapping devices for the blind on the market at present, some with significant drawbacks. Devices can be heavy and cumbersome, which is very problematic in a device intended for extended periods of use. Many of these devices are highly visible, advertising the user's disability. The devices may compromise one or more senses in the process of conveying information, a critical disadvantage for visually impaired users. Many current aids use vibrating buttons or pads in the display to warn of upcoming obstacles, a method which is only capable of conveying limited information regarding direction and proximity to the nearest object. Some of the more sophisticated devices use an audio interface in order to deliver more complex information, but this compromises the user's hearing, a critical impairment for a blind user.

Many currently available orientation devices suffer from lack of accuracy. They often have a limited means of 'mapping' the terrain ahead, and more importantly, they are typically incapable of transmitting/transferring that information usefully to the user. Although many mobility aids can warn of obstacles up to six metres ahead and crudely convey the distance of said objects to the client, they cannot convey what would normally be regarded as field of view information to the user without compromising other critical sensory channels.

Although complex GPS systems have had some success in addressing this limitation, they seldom warn of obstacles immediately ahead, are often unsuited for indoor use, may be extremely bulky to wear, typically are prohibitively expensive and they too often severely compromise the natural function of the auditory sense. They cannot be regarded as stand-alone systems.

If the client is presented with limited orientation feedback, not only is quality of life impaired, but also mobility may be reduced to an isolated step-by-step cane assisted progression, typically punctuated by non specific on/off warning signals from a mobility aid. Relatively few visually impaired people accept the devices that are currently available. This is not surprising as the performance of these devices, for the reasons discussed above, cannot always justify the price tag. Clients will accept the standard Long Cane for its simplicity and 
predictability and the fact that it is approximately a fiftieth of the cost of a sophisticated electronic aid.

\section{COMPETITIVE LANDSCAPE}

Current products have largely not gained significant traction in the market. Some of this is due to an inadequate feature set, sometimes combined with a high retail price. The companies responsible for the competing products tend to be small. There is no one player with a significant market advantage against the others. A typical example is Sound Foresight, a spin-out company from Leeds University which sells the Ultracane (See Fig. 1).

The UltraCane is essentially an advanced, ultrasonic device integrated into a cane [1]. It feeds information about upcoming obstacles through to a series of vibrating buttons on the handle, conveying distance and rudimentary height information. It has two ranges, three metres and five/six metres, and its sensors detect from 1 inch off the floor to 'just above your head'. Since its launch in 2004, Sound Foresight has sold UltraCanes into 15+ countries. It has been featured on television programmes and in newspapers and magazines around the world, and won numerous awards.

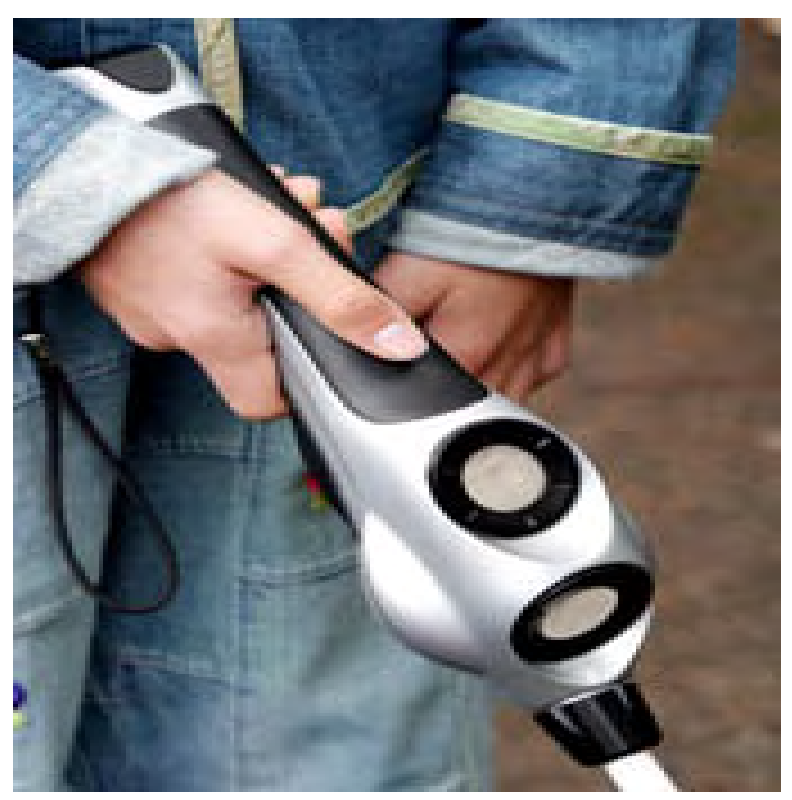

Fig. 1. The Ultracane. Here, the ultrasonic transceiver circuit and user display are appropriately and unobtrusively moulded into the handle of a functional cane. A big step in the right direction! The warning signals are displayed on a vibrating tactile interface. (Photo courtesy of Sound Foresight BatCane)

The K Bat-Sonar (See Fig. 2) takes complex echoes as return signals from ultrasonic waves, initially generated by the device, then translates them into audible tone rich sounds. These synthetic sounds are amplified and sent to earphones worn by the user. When the system is attached to a long cane, it can be used in the usual way by scanning repeatedly from one side to the other. However, the range of the cane is extended beyond the usual short stick length to the range of the transceiver unit clipped on near the handle and which, in fact, becomes the replacement handle for the combined assembly. The system is described as a spatial sensor using echolocation bio-acoustic technology. The handbook describes this as 'sonocular perception'. However, it also refers to the substantial learning commitment required for this conversion to an alternative perception. 'Learning the many subtle nuances of spatial perception is a continuous self-oriented process and extends over a long period of time'.

The statement, ' $\mathrm{K}$ ' Sonar acts as a vision substitute', needs to be examined carefully. There is also a clear suggestion that 'two are better than one' and that the device be used in conjunction with a Longcane [2]. If it were in fact a true substitute for vision, surely there would be no need for attaching it to a cane and relying on the cane as a primary close range assistive device? It is true that the BAT website [2] does admit limitations of both cane and device; 'This combination removes most of the limitations of either aid by itself.' If we accept this, then must the Longcane also be regarded as a vision substitute?

The Miniguide (See Fig 3) uses ultrasonic echo-location to detect objects [3]. The aid vibrates to indicate the distance to objects - the faster the vibration rate the nearer the object. There is also an earphone socket which can be used to provide sound feedback. A single push button is used to switch the aid on or off and also change settings. The aid can accommodate ranges of between $0.5 \mathrm{~m}$ and $8 \mathrm{~m}$, depending on the chosen mode. The Miniguide has got a transmitter/ receiver pair that should be held one above the other while in operation. Thus, users must pay attention to ensure they are holding their devices vertically. This, we believe, inhibits 'unconscious' operation.

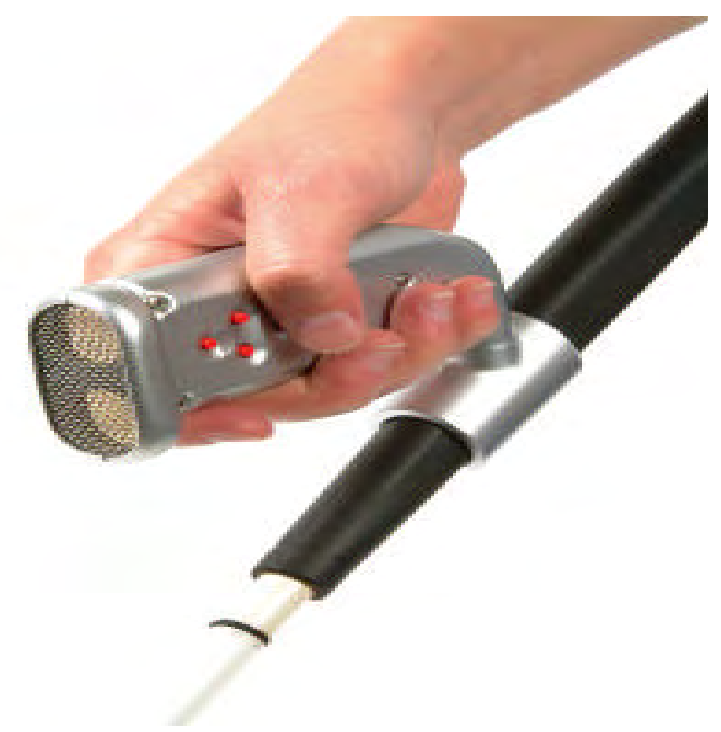

Fig. 2. BAT ' $K$ ' Sonar. This device is likened to a flashlight that sends out silent ultra high frequency sound signals. The signals bounce off obstacles in the path of the device, returning to the receiver sensor. The unit translates these signals into audible 'tone complex' sounds which are then amplified and sent to the user's earphones. The system can be added to a standard long cane. (Photo courtesy of Bay Advanced Technologies Ltd.) 


\section{LIMITED ACCEPTANCE OF DEVICES}

There are a number of reviews such as those listed in Currently Available Electronic Travel Aids for the Blind [4]. None of these can be regarded as more than a rough guide. Clear evidence of why current aids are rejected can be found in relevant conference and journal papers such as $[5,6,7]$. Blasch for example, states that few are regularly used. Davies in 2006 refers to only limited continued use of the device [6].

A specific reference is made to the Sonic Pathfinder (See figure 4), one of more expensive and complicated systems. The downfall of many of current devices is that they prioritise the obstacle immediately in front of the user and do not provide additional information to the user. ETA rejection has existed since the report from National Research Council [8]. This report refers to auditory interfaces that compromise the natural feedback derived from tapping a long cane. These auditory displays are still the most common user interface in more sophisticated orientation devices.

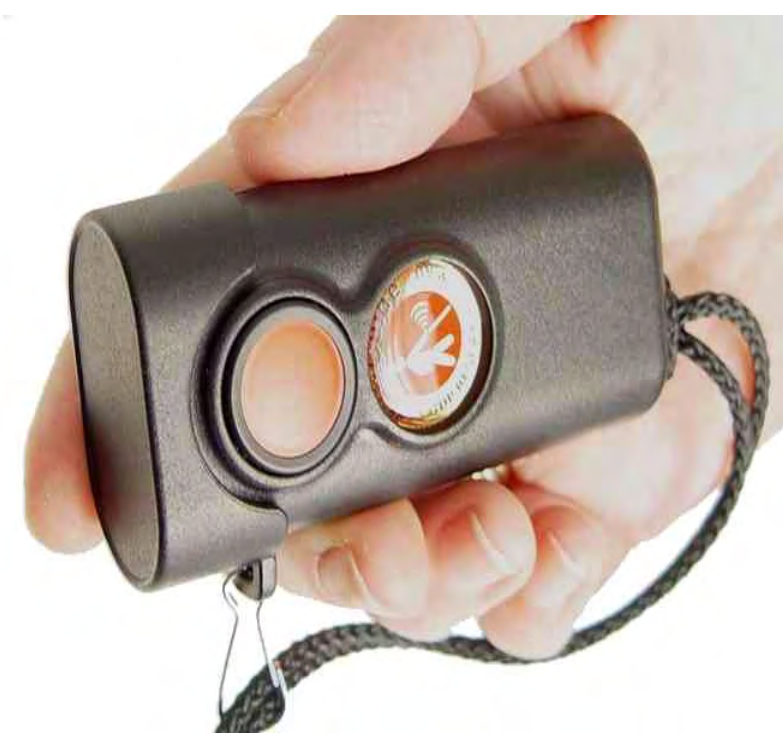

Fig. 3 The Miniguide is a small handheld device that uses ultrasonic pulses to echo locate obstacles in its path. It has the advantage of a low current requirement. However, when used indoors, most ultrasonic devices pick up unwanted ambient echoes from adjacent walls, ceilings and surfaces which may corrupt the result. Vibration displays usually draw more current than the rest of the circuit requirement. (Photo courtesy of GDP Research)

The Teletact 2, in order to overcome some of the problems associated with both laser telemeter and infra-red forward scanning technologies, combines both in one system [9]. An earlier version made use of the laser only, the reflected beam of which can result in a confused signal from plate glass, such as in a door or front to a building. There was also a problem with lasers not picking up dark objects, such as black cars or other vehicles. Grass at the side of a path could also be confusing to a laser-based system.

In case of both proximeter and laser telemetric detection, the system transmits telemeter information. When it senses the proximeter signal only, it sends a "window warning" signal to the user, in order to warn them that they may be approaching a window. The proximeter works within a range of 3 meters, and gives a window pane / black car detection up to two meters.

It uses vibrating devices located under the user's fingers. Experiments were conducted with two, four and eight vibrating devices, and the four-device solution turned out to be the most successful. The principle of this method is simple. Each finger (except the thumb) is in contact with one and only one vibrating pad. Each vibrating pad corresponds to a distance interval. If an obstacle is detected within one of the four distance intervals, then the corresponding vibrating device is activated.

Although the Teletact 2 overcomes some of the problems associated with the previous model, it is essentially a go/no go device. The design is unique in that it makes use of both an infra-red proximeter and laser telemeter. Infra-red systems usually work well indoors, but can be adversely affected by interference from the outdoor environment, such as sunlight.

The Sonic Pathfinder is a head mounted device. This system evolved from work of The Blind Mobility Research Unit at Nottingham University. It is designed for out of doors use in conjunction with a Longcane, a dog or residual vision [10]. The system is a head-mounted pulseecho sonar system incorporating five transducers and a microcomputer. The main decision algorithm reacts to the nearest object and is center weighted, displaying earphone tones on a pitch-to-distance rationale. Many sonar-based systems do not function well inside walled areas due to false echoes confusing valid return data.

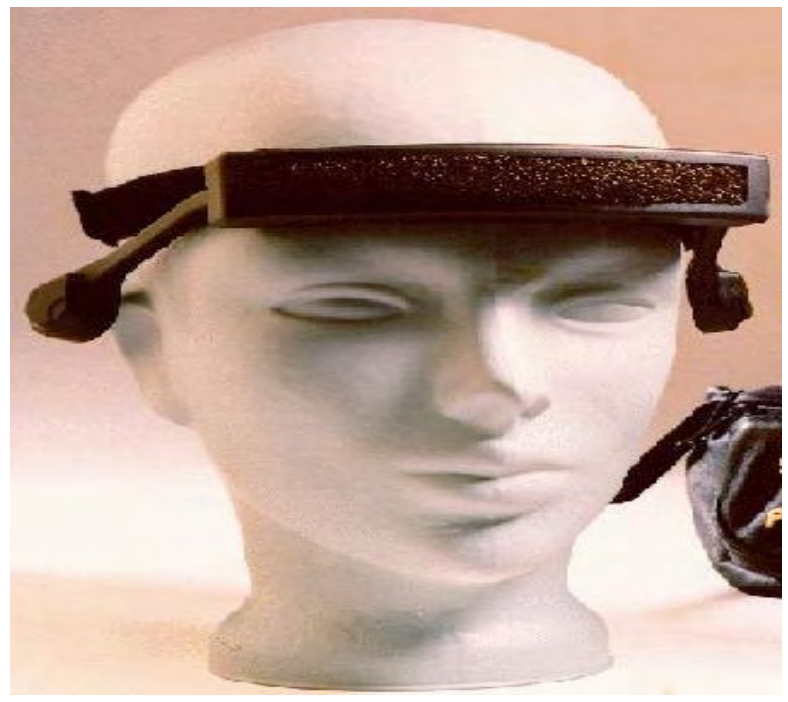

Fig 4. Sonic Pathfinder. The headband contains five ultrasonic transducers, three receivers and one transmitter. Echoes from transmitted signals trigger a range of distance alert tones, which are delivered through earphones to the user. (Photo courtesy of Perceptual Alternatives) 


\section{ESCALATION IN DEMAND}

In the developed countries the number of blind people was estimated to be 3.5 million in 1990 and 3.8 million in 2002, an increase of $8.5 \%$.

\section{Australia}

About 480,000 of Australia's 20 million residents are visually impaired, and over 50,000 of these people are legally blind. Projections indicate that by 2024, over 800,000 Australians will suffer from visual impairment, and approximately 90,000 will be blind [11].

\section{America}

The total number of Americans with blindness in 1995 was approximately 1.3 million, and that number grew to 1.5 million in 2000. Incorporating the high death rates for older age groups, the expected net growth in the prevalence of low vision and blindness is approximately 36,000 cases per year until 2025. However, the annual incidence, the number of new cases added each year, will grow from the current 256,000 to 500,000 in 2020 [12].

\section{Globally}

The World_Health Organization estimated that in 2002 there were 161 million (about $2.6 \%$ of the world population) visually impaired people in the world, of whom 124 million (about 2\%) had low vision and 37 million (about $0.6 \%$ ) were blind [13].

In developing countries, excluding China and India, 18.8 million people were blind in 1990 compared to 19.4 million in 2002, an increase of $3 \%$. In China and India the estimated numbers of blind people in 1990 were 6.7 and 8.9 million, respectively; in 2002 there were an estimated 6.9 million blind people in China and 6.7 million in India. These figures indicate an increase of $3 \%$ in the number of blind people in China and a decrease of $25 \%$ in India.

The following is a quote from Margrain [14]:

"The number of people with impaired sight that cannot be improved with the use of spectacles or other treatments is growing. Demographic data suggests that the numbers of people with impaired vision are likely to increase at least until 2021 because the main causes of low vision are age related. Medical intervention is unlikely to reduce significantly the numbers of people with impaired vision in the foreseeable future because there is currently no treatment for the primary cause of visual impairment, age related macular degeneration. Given that it will not be possible to cure visual impairment the emphasis must be on providing an effective rehabilitative low vision service."

Client statistics from the Canadian National Institute for the Blind (CNIB) show an increase in those in need of services from their organization; and these numbers are considered to be conservative because data collection is a result of self-report and collected from individuals who participate in their services.

Vision impairment is responsible for 18 percent of hip fractures by older Americans at a cost of treatment of \$2.2 billion each year. If we could prevent just 20 percent of such hip fractures, it is estimated that US\$441 million would be saved annually [15]. This is just one example of the considerable healthcare costs caused by vision impairment.

\section{DEVICE CATEGORISATION}

Existing devices can be broken down into two categories. First the simpler type that warn of an obstacle in the forward vicinity of the user, but convey little or no detail with respect to position or object identification. They may use buzzers, simple warning vibration or synthetic tones as the user interface. They do not usually warn of drop-offs, such as potholes, in any truly reliable way.

The second category may have enhanced range and precision, as in the case of some laser based types, but often with a far too simplistic binary information go/no go user interface, or, alternatively, use complex sonar sweeping techniques that convert ultrasonic reflected signals into a synthetic but inhuman audio signal that is presented to the user. Such devices require substantial learning and compromise the natural sound cues that are absolutely essential for a blind person.

Many of the competing products have poor and inappropriate human-machine interfaces. A recent paper in the Proceedings of the 2005 IEEE Engineering in Medicine and Biology Conference reinforces these views [16]. Velazquez et al confirm that although many ETAs have been proposed to improve mobility and safety navigation independence for the visually impaired, none of these devices is widely used and user acceptance is low. Four shortcomings are identified in all ETAs.

1. They obtain a $3 D$ world perception via complex and time-consuming operations: environment scanning using sonar-wave or laser beam requires the user to actively scan the environment, to memorize the gathered information, to analyze it and to take a decision: constant activity and conscious effort that requires intense concentration, reduces walking speed and quickly fatigues the user.

2. They provide an acoustic feedback that interferes with the blind persons ability to pick up environmental cues. Another problem is degradation and overloading of the hearing sense. Most of these critical interfaces are designed by electronics engineers who have little knowledge of human perception. Many of these devices had their origins as robotics projects.

3. They are invasive. They are intrusive and disturb the environment with their scanning and feedback technologies.

4. They are still burdensome and conspicuous to be portable devices, which are essential needs for people with visual impairments.

Hakkinen's IEEE conference paper [17] refers directly in the title to 'Postural Stability and Sickness Symptoms After Head Mounted Display Use.' The findings show clearly these common displays produce adverse affects on the user. 


\section{SOUND SUBSTITUTION INTERFACES}

Scanned objects normally produce multiple echoes, translated by the receiver into unique invariant 'tonecomplex' sounds, which users listen to and learn to recognize. The human brain is very good (it is claimed) at learning and remembering certain sound-signature sequences in a similar way that it learns a musical tune. The sound signatures vary according to how far away the device is from the object, thus indicating distance. The user listens to these sounds through miniature earphones and can detect the differences between sound sequences thus identifying the different objects. This allows limited mapping and orientation for the user at a price.

Any auditory user interface has the potential to interfere with the users' hearing of natural ambient sound cues. This is a critical factor for a blind user. If used in a safe environment by a truly driven person prepared to learn over time, sound signatures representing a visual scene could significantly enhance quality of life. However, the 'real world' is not safe, and there are serious safety concerns about restricting the hearing of a blind user in an uncontrolled environment.

Beyond the safety aspect, blind users have learned to depend on their hearing, and any product which continuously interferes with it may lead to a compromised alternative human sensory input. Supporting evidence for this claim can be universally found from very different disciplines. Some of these have already been referenced in the preceding sections. More specific reference can be found from Johnson and Higgins who refer to visual auditory substitution taxing a sensory modality that is already extensively used for communication and localization [18]. Velazquez [16] refers to four shortcomings of existing ETAs. One of these is They provide an acoustic feedback that interferes with the blind person's ability to pick up environmental cues through hearing. Familiar cues may be learned over many years.

Recent studies indicate that a 20 minute usage of acoustic feedback devices causes serious human information registration, reduces the capacity to perform usual tasks and affects the individual posture and equilibrium [17]. Such interfaces may fail because of their complex, confusing and restrictive masking audio feedback, particularly to the frail user. They are often not suitable for a typical elderly blind user who is likely to have multiple disabilities. A Study by Ross and Blasch [19] clearly indicated that blind people preferred a tapping tactile interface to sound feedback.

\section{INTRUSION OF SOUND-BASED DISPLAYS}

Head mounted displays usually incorporate sound feedback which has already been covered. They are also often bulky, relatively heavy and are frequently rejected by the typical user [16 and 17]. Velazquez [16] refers to them as follows:

They are invasive. They are intrusive and disturb the environment with their scanning and feedback technologies. The last thing a blind person needs is further advertisement of his/her disability.

\section{SOLUTION PROTOTYPE DEVELOPMENT}

The author has developed a system, which it is hoped, will be the first step in addressing some of the listed problems. This working prototype has a unique tactile interface design which, even in its basic form, has distinct user advantages over many other systems, including those devices with tactile interfaces. As with some of the sonar systems listed above in the paper, this first prototype is best suited to outdoor use. Future models are not limited to sonar technology, however.

An attempt is being made to address a number of the problems of existing devices listed above in this paper. Some of the criticism found in papers with respect to other devices, could also in some respects, be directed towards this first prototype. It will therefore form the test-bed for a range of better and more advanced designs.

The design criteria has and will in the future, concentrate on intuitive interfaces that do not compromise certain other all-important sensory channels. These interfaces, now under development, will be configurable for users who are both deaf and blind.

There will also be an emphasis on ease of learning and use. It is unacceptable to expect someone, who may have multiple disabilities, to undertake a long and complex learning programme in how to use a new device.

The question of size and weight is also being given top priority for field testing prototype development.

However, no design of mine or of this team, will ever be able to produce a true substitute for human vision, in all its complexity.

\section{CONCLUSION}

Assistive device operation should not impair any other sense. Hearing is all important to the blind user.

There has been considerable resistance demonstrated to products that 'advertise' a user's disability.

Unlike many other devices an ideal system should be configurable for both blind and deaf/blind people.

Although this has yet to be fully quantified, it has been estimated from our own prototype and commercialization market research, that a simple, viable and competitive device could be produced for under $\$ 300$ from standard off-shelf components. The cost of many current products is still too high.

As most existing devices are produced by small, unlisted companies, there is little in the way of publicly available, reliable sales figures, and as such the addressable market is not well defined. However, interviews conducted with industry experts, in addition to the small size of the companies themselves, suggest that these competing devices have so far failed to achieve any significant market presence.

Burns states that a device should ideally be picked up and used immediately. It is often the tedious learning procedures, amongst the other human factors issues listed in this paper, that are commonly the downfall of acceptance of these devices [20].

The aim should be to retain as far as possible, those learned schemas that the user is comfortable with, but at 
the same time extend the possibilities of range and resolution by using the latest appropriate technology. Taking the users background experience into account should be one of the major considerations of a good design; a characteristic that is sometimes neglected in current products.

The author's prototype development programme is, hopefully, a step in the right direction. Further prototype solutions will follow. It is hoped that the description of this novel design and development work may be covered in detail in papers in the near future.

\section{REFERENCES}

[1] UltraCane, http://www.batcane.com

[2] 'K' Sonar, http://www.batforblind.co.nz/how-ksonar-works.php

[3] GDP Research [2003], Miniguide Home Page, http://www.gdp-research.com.au/ultra.htm

[4] Y. Duen, "Currently Available Electronic travel Aids For The Blind" 2007. [Online] www.noogenesis.com.eta/current.html

[5] B. Blasch, results of A National Survey of Electronic Travel Aid Use. Journal of Visual Impairment and Blindness 83, pp 449-453. 1999.

[6] T. Davies, C. Burns and S Pinder, "Using Ecological Interface Design to Develop an Auditory Interface for Visually Impaired travelers," Proc. Of OZCHI 2006, Sydney, Australia. 2006

[7] K Young-Jip, K. Chong-Hui and K. Byung-Kook, “ Design of Auditory Guidance System For The Blind With Signal Transformation from Stereo Ultrasonic to Binaural Sound," Proc of $32^{\text {nd }}$ ISR (International symposium on Robotics), April 2001.

[8] Committee on Vision "Electronic Travel Aids: New Directions For research," Working group on Mobility Aids For The Blind, National research Council, pp 74, National Academy press, Washington, DC 1986.

[9] C.Jacquet et al. (2006): Electronic Locomation Aids for the Blind: Towards More Assistive Systems, Studies in Computational Intelligence (SCI) 19, 133-163.

[10] Sonic Pathfinder, http://web.aanet.com.au/heyes/

[11] Vision 2020: the Right to Sight 1999 - 2005 p.19

[12] Bulletin of the World Health Organization, p.847, Nov. 20

[13] World Health Organization: Magnitude and causes of visual impairment, p.3, Nov 2004.

[14] Margrain, TH. (2000). Helping blind and partially sighted people to read: the effectiveness of low vision aids. British Journal of Ophthalmology. 84, 919-921.

[15] Vision Rehabilitation: Evidence-Based Review, p.25, May 2005.

[16] Velazquez, E. Pissaloux and F. Maingreaud, "Walking Using Touch", Proc. Of 2005 IEEE Engineering in Medicine and Biology $27^{\text {th }}$ Annual Conference, Shanghai, China, 2005.

[17] J. Hakkinen, "Postural Stability and Sickness symptoms After HMD Use", Proc. Of IEEE International conference on Systems, Man and cybernetics, Hammamet, Tunisia, 2002.

[18] L Johnson and C Higgins, "A Navigation Aid for the Blind using Tactile-Visual Sensory Substitution," Department of Electrical and Computer engineering program, university of Arizona, Tucson USA. 2006.

[19] D. Ross and B. Blasch, "Wearable Interfaces for orientation and wayfinding," Proc. Of ASSETS 2000, Arlington, Virginia, USA. 2000.

[20] C. Burns and J Hajdukiewiez, "Ecological Interface Design," Florida: CRC Press, pp 4, 2004. 